\section{ORIGINAL} RESEARCH

F.B. Mohamed

L.N. Hunter

N. Barakat

C.-S.J. Liu

H. Sair

A.F. Samdani

R.R. Betz

S.H. Faro

J. Gaughan

M.J. Mulcahey

\title{
Diffusion Tensor Imaging of the Pediatric Spinal Cord at 1.5T: Preliminary Results
}

BACKGROUND AND PURPOSE: Recent studies suggest that pediatric subjects as old as 8-years-of-age may have difficulty with the ISNCSCI examinations. Our aim was to investigate DTI parameters of healthy spinal cord in children with noncervical IS for comparison with children with $\mathrm{SCl}$ and to prospectively evaluate reliability measures of DTI and to correlate the measures obtained in children with $\mathrm{SCl}$ with the ISNCSCl.

MATERIALS AND METHODS: Five controls with thoracic and lumbar IS and 5 children with cervical SCI were imaged twice by using a single-shot echo-planar diffusion-weighted sequence. Axial imaging was performed to cover the entire cervical spinal cord in controls. For the SCI subjects, 2 vertebral bodies above and below the injury were imaged. FA and $\mathrm{D}$ values were obtained at different levels of the cervical spinal cord. All subjects with $\mathrm{SCl}$ had undergone ISNCSCI clinical examinations. Statistical analysis was performed to access differences of the DTI indices between the controls and SCl subjects, reproducibility measurements, and correlations between DTI and ISNCSCI.

RESULTS: Subjects with SCl showed reduced FA and increased D values compared with controls. Test-retest reproducibility showed good ICC coefficients in all the DTI index values among controls $(\geq 0.9)$, while the $\mathrm{SCl}$ group showed moderate ICC $(\geq 0.77)$. There were statistically significant correlations between the various DTI indices and ISNCSCI scores.

CONCLUSIONS: Preliminary DTI indices in children were determined and showed good reproducibility. Reduced FA and increased D values were seen in children with $\mathrm{SCl}$ in comparison with controls and showed good clinical correlation with ISNCSCI examinations.

\begin{abstract}
ABBREVIATIONS: $A C=$ anal contraction; $A I S=A S I A$ impairment scale; $A S=$ anal sensation; $\mathrm{ASIA}=$ American Spinal Injury Association; $\mathrm{Cl}=$ confidence interval; $\mathrm{D}=$ diffusivity; $\mathrm{DTI}=$ diffusion tensor imaging; $F A=$ fractional anisotropy; $F A C T=$ fiber assignment by continuous tracking; ICC = intraclass correlation; IS = idiopathic scoliosis; ISNCSCI = International Standards for Neurological Classification of SCl; LT = light touch; $\mathrm{ML}=$ motor level; $\mathrm{MS}=$ motor score; $\mathrm{NL}=$ neurologic level; $\mathrm{PP}=$ pin prick; $r_{\mathrm{s}}=$ Spearman correlation coefficient; $\mathrm{SCl}=$ spinal cord injury; $\mathrm{SL}=$ sensory level; TD = typically developing
\end{abstract}

$\mathbf{T}$ he ISNCSCI are currently used to determine and classify the extent of motor and sensory impairment following SCI. The ISNCSCI involve strength testing of 10 key muscles; testing of sensory appreciation to LT and sharp/dull discrimination in 56 dermatomes; and sensory and motor testing of the lowest sacral segments, including an internal rectal examination. ${ }^{1}$ The ISNCSCI is used to classify the consequence of SCI, access neurologic recovery, and determine eligibility for participation in clinical trials. Recent reports suggest that the ISNCSCI examinations do not have utility for children younger than 6 years of age and that some children as old as 8

Received April 13, 2010; accepted after revision June 30

From the Department of Radiology (F.B.M., N.B., C.-S.J.L., S.H.F.) and Biostatistics Consulting Center (J.G.), Temple University School of Medicine, Philadelphia, Pennsylvania; Shriners Hospitals for Children (L.N.H., A.F.S., R.R.B., M.J.M.), Philadelphia, Pennsylvania; and Department of Radiology (H.S.), Massachusetts General Hospital, Boston, Massachusetts

The study was funded by the Shriners Hospitals for Children, grant 8956. (M.J.M., Principal Investigator).

Paper previously presented in part at: Annual Meeting of the American Society of Neuroradiology, May 16-19, 2009; Vancouver, British Columbia, Canada; and Annual Meeting of the Radiological Society of North America, November 29-December 3, 2009; Chicago, Illinois.

Please address correspondence to Feroze B. Mohamed, PhD, Department of Radiology, 3401 N Broad St, Temple University School of Medicine, Philadelphia, PA 19140; e-mail: feroze@temple.edu

DOI 10.3174/ajnr.A2334 years of age may have difficulty with the examinations. ${ }^{2}$ Also, studies on the repeatability of anorectal examinations show poor-to-moderate agreement, ${ }^{2,3}$ with some questioning their validity as an index of cord injury severity. ${ }^{1,4}$ More important, while the ISNCSCI are widely used, they are a proxy assessment of the integrity of the spinal cord because they involve clinical examination of motor and sensory function of the limbs, trunk, and anorectal area rather than a direct objective evaluation of the integrity of spinal cord tracts.

DTI is a relatively new noninvasive MR imaging technique that quantifies the diffusion of water molecules in directions parallel and transverse to the axis of neuronal axons. In the spinal cord, the axons and myelin sheaths in the white matter are oriented longitudinally; thus, maximum water diffusion occurs in this direction. In gray matter, this type of axonal organization is lacking. Thus, DTI can differentiate white matter structures of high and low anisotropy, quantify directionality of the white matter tracts, provide quantification of 3D water diffusivity, and track healthy nerve tissue within white matter.

While the utility of DTI has been demonstrated for intracranial pathologies, ${ }^{5}$ initial studies documenting its usefulness in the spinal cord have been reported. Preclinical studies suggest that DTI is useful in determining motor recovery following rat SCI and treatment. ${ }^{6,7}$ Human studies also demonstrate the promise of this technology. ${ }^{8-11}$ How- 
ever, most the human spinal cord studies involve adult subjects.

If DTI can be a reliable method for quantifying viable neural tissue within the spinal cord in young children, it may be a useful neurodiagnostic adjunct to clinical measurement and/or conventional MR imaging. Children with SCI routinely receive a standard MR imaging of the spine as a follow-up to their injury, and a DTI scan could easily be performed during the same session, only requiring approximately an additional 8 minutes of session time.

Because there are no published reliability data on the DTI technique in children of any age with SCI and because children younger than of 6 years of age cannot participate in the ISNCSCI examination, it is necessary to first demonstrate the correlations between the DTI technique and ISNCSCI scores in older children, in whom the ISNCSCI examination can be reliably administered. Once reliability and correlations are established in the older age group, one can then develop and test a method to evaluate and classify the neurologic consequence of SCI in children younger than 6 years of age by using the DTI technique as a validation tool. Thus, the purpose of this prospective pilot study was 3-fold: 1) to investigate DTI parameters of healthy spinal cord in children with thoracolumbar (noncervical) IS as a means of comparing them with children with SCI, 2) to evaluate the reliability and validity of DTI in children, and 3) to evaluate how well DTI compares with clinical neurologic variables obtained in children with SCI with the ISNCSCI.

\section{Materials and Methods}

\section{Study Design and Sample}

The study used a cross-sectional repeated-measures design involving a sample of convenience from a single site. Ten subjects, 5 with noncervical IS without evidence of spinal cord pathology (mean age, 15.2 years) served as controls, and 5 with SCI (mean age, 11.6 years) were enrolled in this study. Subjects with SCI were excluded from the study if the following conditions were present: 1) They had sustained a traumatic brain injury at time of the SCI, resulting in the inability to follow test instructions; 2) they had sustained a high-level injury that required mechanical ventilation without a means to communicate their responses to clinical testing; or 3) they had a mental health status that may be exacerbated by clinical testing of their injury (suicidal ideation). Subjects and their parents provided written informed assent and consent, respectively, of the protocol approved by the institutional review board. All subjects underwent MR imaging twice within 4-5 weeks, and subjects with SCI had complete motor, sensory, and anorectal examinations that were performed by a trained rater on the basis of the current ISNCSCI techniques. ${ }^{1,12}$

\section{Clinical SCI Protocol}

As per the ISNCSCI, each subject with SCI had testing of 28 dermatomes on the right and left side of the body for sensitivity to sharp/ dull discrimination (via PP testing and LT). At a specified point in each dermatome, sensitivity to PP and LT was evaluated and scored by using the 3-point scale consisting of 0 (absent), 1 (impaired), and 2 (unimpaired). Summed sensory scores were calculated for the rightsided PP and LT and left-sided PP and LT. Total PP and LT scores were defined by summing the right and left PP and LT scores, respectively. The sum of the total PP and LT scores resulted in the single sensory score. Subjects also had a motor examination that was completed through the testing of the strength of 10 key muscles bilaterally.

The strength of each muscle was graded on a 6-point ordinal scale between 0 (complete paralysis) and 5 (normal active movement, full range of motion against full resistance). These scores were summed across myotomes and sides of the body to generate a single total MS. The anorectal examination was performed to evaluate sensation and contraction of the external anal sphincter. For this examination, the examiner applied pressure with the index finger to the rectal wall to test for AS. To test for AC, the person being examined was asked to squeeze the examiner's finger as if holding a bowel movement. Scoring for both AS and AC was dichotomous (yes/no).

The sensory, motor, and anorectal examination scores were used to determine the highest NL, SL, ML, severity of injury (complete/ incomplete), and zones of partial preservation (partially innervated dermatomes and myotomes in the complete injuries only) on the basis of the AIS. ${ }^{1,12}$

\section{MR Imaging Protocol}

The MR imaging protocol consisted of an initial T2-weighted sagittal scan of the entire spinal cord. The sagittal images were used to prescribe axial sections of the spinal cord. Next, conventional axial T2weighted scans were obtained on controls and children with SCI. Finally, DTI images were obtained in the same anatomic location prescribed for the T2-weighted images. The MR imaging parameters for the axial fast spin-echo T2-weighted imaging were as follows: $\mathrm{TR}=3500 \mathrm{~ms}, \mathrm{TE}=124 \mathrm{~ms}, \mathrm{FOV}=240 \mathrm{~mm}, 256 \times 256$, and 2 acquisitions. All the scanning was performed by using a 1.5T Signa scanner (GE Healthcare, Milwaukee, Wisconsin).

DTI was performed by using a single-shot echo-planar diffusion-weighted imaging sequence. The scanner is equipped with a $33-\mathrm{mT} / \mathrm{m}$ gradient amplitude with a gradient rise time of $276 \mu \mathrm{s}$, which is extremely useful for physiologic imaging like DTI. A standard 8-channel phased-array coil was used for scanning. To determine the diffusion tensor fully, we obtained diffusion-weighted images along 6 different directions with a b-value of $700 \mathrm{~s} / \mathrm{mm}^{2}$ as well as an image acquired without diffusion weighting $(b=0$ $\mathrm{s} / \mathrm{mm}^{2}$ ). A slab of DTI acquisition consisted of twenty-three $3-\mathrm{mm}$ axial sections with no intersection gaps. In controls, 2 slabs of DTI images were acquired to cover the entire cervical spinal cord (C1C7). In subjects with SCI, 1 slab of DTI images was acquired with the most central section (section 12) placed in the middle of the injury ( 2 vertebral bodies above and below the injury based on T2 images and confirmed by a neuroradiologist). This was done to reduce patient discomfort by reducing the overall imaging time spent inside the scanner. Other imaging parameters included the following: $\mathrm{TR}=6000 \mathrm{~ms}, \mathrm{TE}=60 \mathrm{~ms}, \mathrm{FOV}=240 \mathrm{~mm}, 128 \times 128$, and 4 acquisitions. The total imaging time to collect 1 slab of DTI images was approximately 8 minutes. Sedation and/or anesthetic was not administered to the subjects in this study. To test reproducibility of the DTI scans, we brought the patients back within a mean of 34.3 days to the MR imaging center and scanned them a second time. During the second visit, care was taken to position the subjects in the MR imaging scanner in the same anatomic location as those in the first visit. This was performed with the aid of the sagittal image from the first scan for controls and subjects with SCI as well as placement of the first section location at the superior margin of the dens of the $\mathrm{C} 1$ vertebral body for controls. 


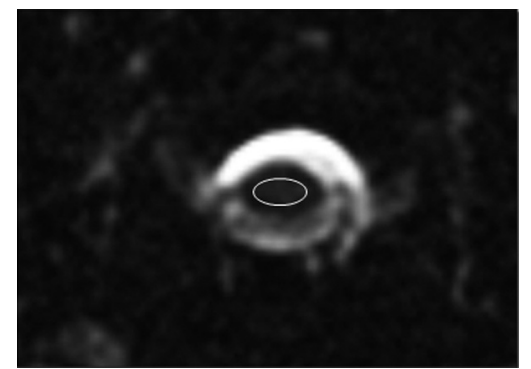

Fig 1. Region-of-interest placement on an axial BO image of the cervical spine used in this study.

\section{Image Processing and Statistical Analysis}

After image acquisition, the data were transferred to an independent workstation in the MR imaging laboratory for calculation of the DTI indices. The diffusion tensor was calculated from the diffusionweighted images on the basis of the method proposed by Basser and Pierpaoli, ${ }^{13}$ by using DTIStudio software (Johns Hopkins University, Baltimore, Maryland). Regions of interest were drawn on individual axial sections (C1-C7) by a board-certified pediatric neuroradiologist for the 2 separate acquisitions (Fig 1). There was a consistent sparing of the outer margin of the cervical cord that represented approximately 1 voxel width to minimize volume averaging with the CSF. Various DTI indices, namely radial (or transverse) D, axial (or longitudinal) D, average D, and FA, were calculated and tabulated. Statistical analysis was performed by a biostatistician to access the significance of the diffusion indices between the 5 controls and 5 children with SCI at all the levels of the cervical spinal cord. The data from the 2 scans were averaged for the statistical analysis. The total number of measured region-of-interest observations was 348 for controls and 239 for children with SCI. Reproducibility of the DTI indices was accessed by using ICC coefficient calculations. We performed $r_{\mathrm{s}}$ to assess correlations between the diffusion indices and ISNCSCI scores in children with SCI. Finally, MR tractography of the spinal cord in a representative control case and a subject with SCI was generated by using the FACT algorithm implemented in Volume-One software (www.volume-one.org). The following parameters were used to generate the tractography: FA threshold of $<0.01$ and stopping angle of $>25^{\circ}$.

\section{Results}

\section{ISNCSCI Scores}

Of the subjects with SCI (Table 1), there were 3 classified as AIS A (complete); 1, AIS B (sensory incomplete); and 1, AIS D (sensory and motor incomplete). The highest neurologic levels ranged from $\mathrm{C} 1$ to $\mathrm{C} 5$, with motor and sensory levels ranging from $\mathrm{C} 1$ to $\mathrm{C} 7$. For all subjects with SCI, LT total scores ranged from 20 to 72 of a possible 112 points (mean, 38.0), total PP scores ranged from 16 to 93 of a possible 112 points (mean 39.2), and total MSs ranged from 26 to 72 of a possible 100 points (mean, 40.8).

\section{MR Imaging Results}

For both the control and subject populations, the DTI values measured across all the axial sections of the cervical spinal cord were averaged.

The control subjects showed an average $\mathrm{FA}=0.62, \mathrm{SD}=$ 0.11 ; average $\mathrm{D}=0.72 \times 10^{-3} \mathrm{~mm}^{2} / \mathrm{s}, \mathrm{SD}=0.17 \times 10^{-3}$; radial $\mathrm{D}=0.44 \times 10^{-3} \mathrm{~mm}^{2} / \mathrm{s}, \mathrm{SD}=0.24 \times 10^{-3}$; and axial $\mathrm{D}=1.23 \times 10^{-3} \mathrm{~mm}^{2} / \mathrm{s}, \mathrm{SD}=0.29 \times 10^{-3}$. The subjects with SCI showed reduced FA values and increased D values compared with control subjects: $\mathrm{FA}=0.39, \mathrm{SD}=0.22$; average $\mathrm{D}=1.27 \times 10^{-3} \mathrm{~mm}^{2} / \mathrm{s}, \mathrm{SD}=0.67 \times 10^{-3} ;$ radial $\mathrm{D}=1.06 \times$ $10^{-3} \mathrm{~mm}^{2} / \mathrm{s}, \mathrm{SD}=0.69 \times 10^{-3}$; and axial $\mathrm{D}=1.65 \times$ $10^{-3} \mathrm{~mm}^{2} / \mathrm{s}, \mathrm{SD}=0.65 \times 10^{-3}$. Significant differences were seen in the average FA $(P<.007)$ (Fig 2$), \mathrm{D}(P<.05)$ (Fig 3$)$, and radial $\mathrm{D}(P<.01)$ (Fig 4$)$ values between the healthy and SCI subjects. However, the axial D measurements (Fig 5) were not statistically significant $(P=.17)$ between the 2 groups. As anticipated, 1 of the subjects classified as AIS D (subject 2) showed FA and D values closer to the normative values; classification of AIS D indicates that the child had sensory and functional preservation that was indicated by DTI.

Figure $6 A$ shows an MR tractography image of the cervical spinal cord of a child with IS derived from FA values in the white matter tracts, a measure of the degree of myelination of the white matter tracts along the spinal cord. This image is different from that of a child with SCI at the C4-C7 levels, which shows a lesser number of tracts below the level of injury (Fig 6C, arrow). However this algorithm (FACT) failed ${ }^{14}$ to track the rest of the cervical cord well below the injury level, even though the FA measurements for subject 4 showed recovery of FA values as seen in Fig 2. A conventional midline sagittal T2-weighted image demonstrated focal atrophy of the lower cervical cord with associated abnormal increased signal intensity involving the mid-C5 though the imaged portion of the upper thoracic spine (Fig 6B), consistent with posttraumatic myelomalacia and gliosis.

Test-retest agreement showed strong ICC in all the control group DTI index values $(\geq 0.9)$ : FA (ICC $=0.90, \mathrm{CI}=0.30$ .99), $\mathrm{D}(\mathrm{ICC}=0.97, \mathrm{CI}=0.73-1.0)$, axial D $(\mathrm{ICC}=0.97$, $\mathrm{CI}=0.70-1.0)$, and radial D $(\mathrm{ICC}=0.94, \mathrm{CI}=0.51-0.99)$. In the group with SCI, test-retest agreement showed fair-tomoderate ICC $(\geq 0.7)$ : FA ( ICC $=0.79, \mathrm{CI}=0.0-0.98), \mathrm{D}$ $(\mathrm{ICC}=0.77, \mathrm{CI}=-0.0-0.98)$, axial $\mathrm{D}(\mathrm{ICC}=0.80, \mathrm{CI}=$ $0.0-0.98)$, and radial D (ICC $=0.77, \mathrm{CI}=0.0-0.98)$ in all the DTI index values measured. As anticipated, the 95\% CIs were wide due to the small number of subjects inherent in pilot studies. There were statistically significant correlations between DTI indices and ISNCSCI clinical impairment scores (Table 2).

\section{MR Imaging-ISNCSCI Correlation}

According to $r_{\mathrm{s}}$ and $P$ values (Table 2) for all subjects with SCI, there was a fairly positive relationship between FA values and left PP scores, manual muscle test scores of the lower extremities, total MS, and AC ( $\left.r_{s}=0.25-0.49\right)$. There was a moderately $\left(r_{\mathrm{s}}=0.50-0.75\right)$ positive relationship between FA values and total/individual (left and right) LT and PP scores for S4-5 dermatomes. In addition, $r_{\mathrm{s}}$ demonstrated a fairly negative relationship between $\mathrm{D}$ and manual muscle test scores of the lower extremities, AC, the highest NL, and total/individual (left and right) LT and PP scores for S4-5 dermatomes. However, the manual muscle test scores of the upper extremities did not follow the positive correlation for FA and negative correlations for D of those ISNCSCI scores that were found to be significant $(P<.05)$. 


\begin{tabular}{|c|c|c|c|c|c|c|c|c|c|c|c|}
\hline $\begin{array}{l}\text { Subject } \\
\text { No. }\end{array}$ & $\begin{array}{r}\text { Age } \\
\text { (yr) }\end{array}$ & AIS & NL & $\mathrm{ML}$ & SL & Severity & $\begin{array}{l}\text { Total LT } \\
\text { Score }\end{array}$ & $\begin{array}{l}\text { Total PP } \\
\text { Score }\end{array}$ & $\begin{array}{l}\text { Total } \\
\text { MS }^{\mathrm{b}}\end{array}$ & AS & $A C$ \\
\hline SCI1 & 8 & $B$ & $\mathrm{C} 1$ & $\mathrm{C} 1$ & $\mathrm{C1}$ & Incomplete & 36 & 32 & 34 & Yes & No \\
\hline $\mathrm{SCl} 2$ & 14 & D & C2 & C2 & C2 & Incomplete & 72 & 93 & 72 & Yes & Yes \\
\hline $\mathrm{SCl} 3$ & 15 & A & C4 & $\mathrm{C7}$ & C4 & Complete & 20 & 16 & 29 & No & No \\
\hline SCI4 & 9 & A & C5 & C6 & C5 & Complete & 33 & 31 & 26 & No & No \\
\hline SCl5 & 12 & A & C5 & C5 & $\mathrm{C7}$ & Complete & 29 & 24 & 43 & No & No \\
\hline
\end{tabular}

a Maximum score, 112 points.

${ }^{\mathrm{b}}$ Maximum score, 100 points

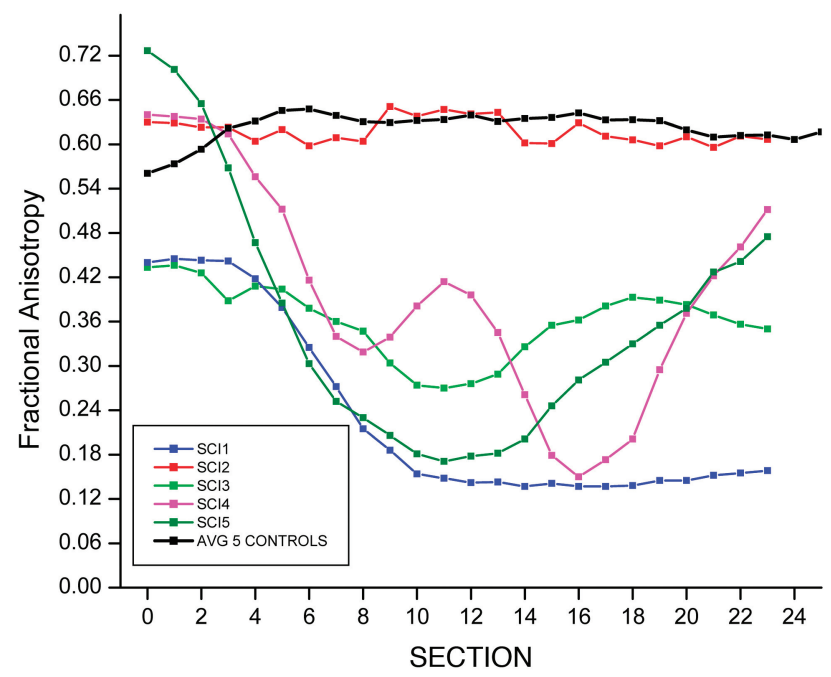

Fig 2. Average FA values for controls compared with FA values for each individual subject, with $\mathrm{SCl}$ as a function of section number.

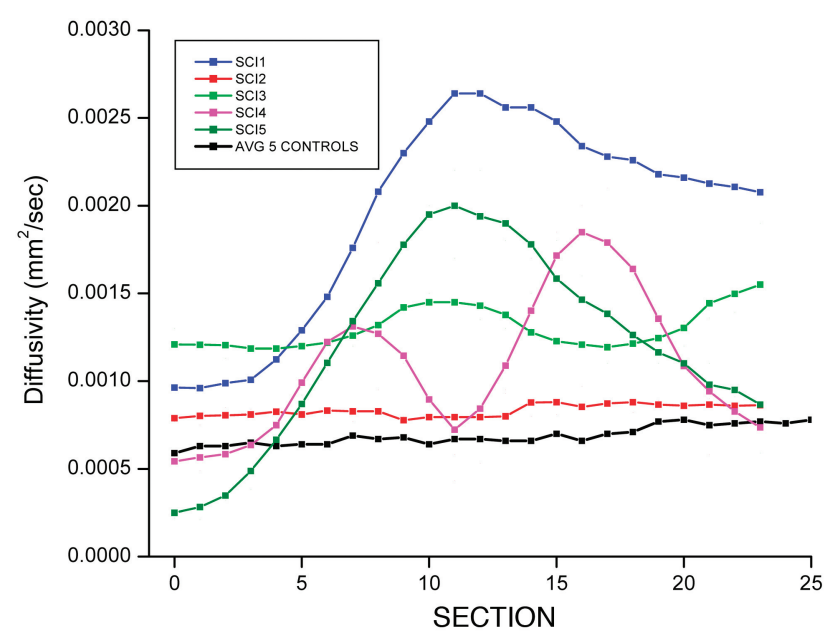

Fig 3. Average $D$ values for controls compared with $D$ values for each individual subject, with $\mathrm{SCl}$ as a function of section number.

\section{Discussion}

Development of a quantitative and reliable method to evaluate injury to the spinal cord at the cord level is essential. This is particularly important for children and others (patients with traumatic brain injury, coma, and so forth) who cannot adequately participate in ISNCSCI, which is currently used for clinical treatment plans, prognostication of outcomes, appropriate placement into clinical research trials, and determination of treatment effectiveness. This study is an initial step toward establishing a reliable and valid quantitative method to evaluate and classify the neurologic consequences of SCI in

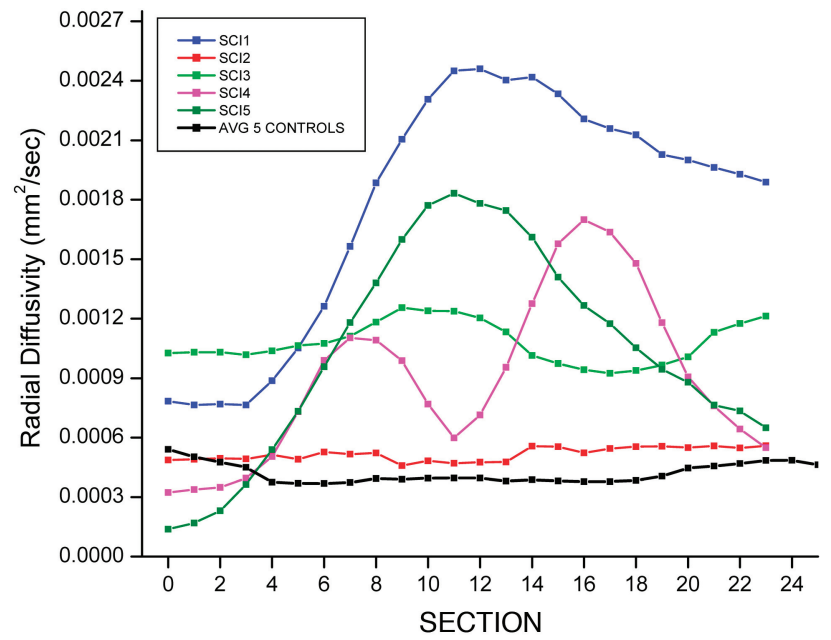

Fig 4. Average radial $D$ values for controls compared with radial $D$ values for each individual subject, with $\mathrm{SCl}$ as a function section number.

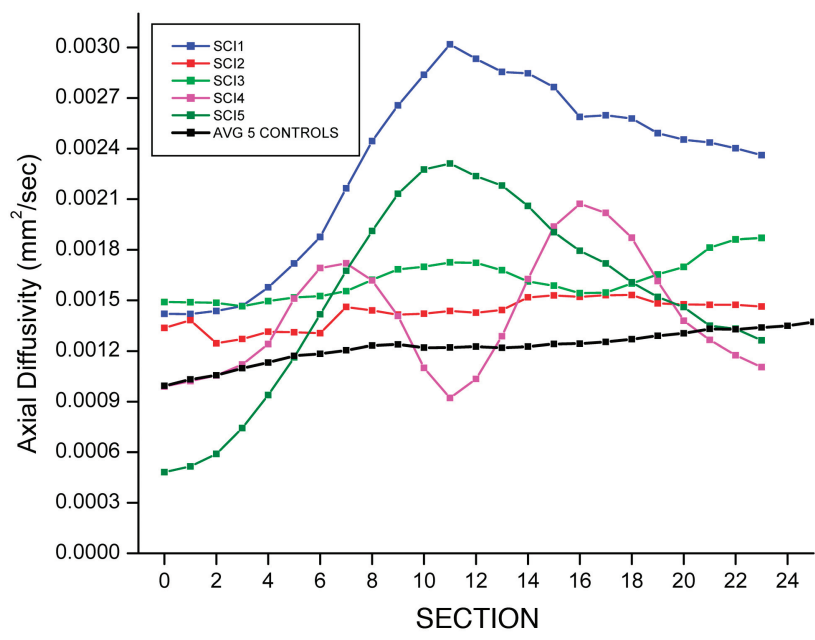

Fig 5. Average axial $D$ values for controls compared with $D$ values for each individual subject, with $\mathrm{SCl}$ as a function of section number.

children. The work is needed for a fundamental reason-no method to evaluate and classify the neurologic consequence of SCI in young children currently exists. Without this ability, we cannot reliably determine motor or sensory level deficits or establish the diagnosis of a complete or incomplete injury and prognosticate recovery.

Prior studies ${ }^{1,2}$ have shown unacceptably high disagreement rates among physicians and other health care providers regarding neurologic level and severity of injury in children. Moreover, the validity, a fundamental requirement of measurement, of the anorectal examination as an indicator of in- 

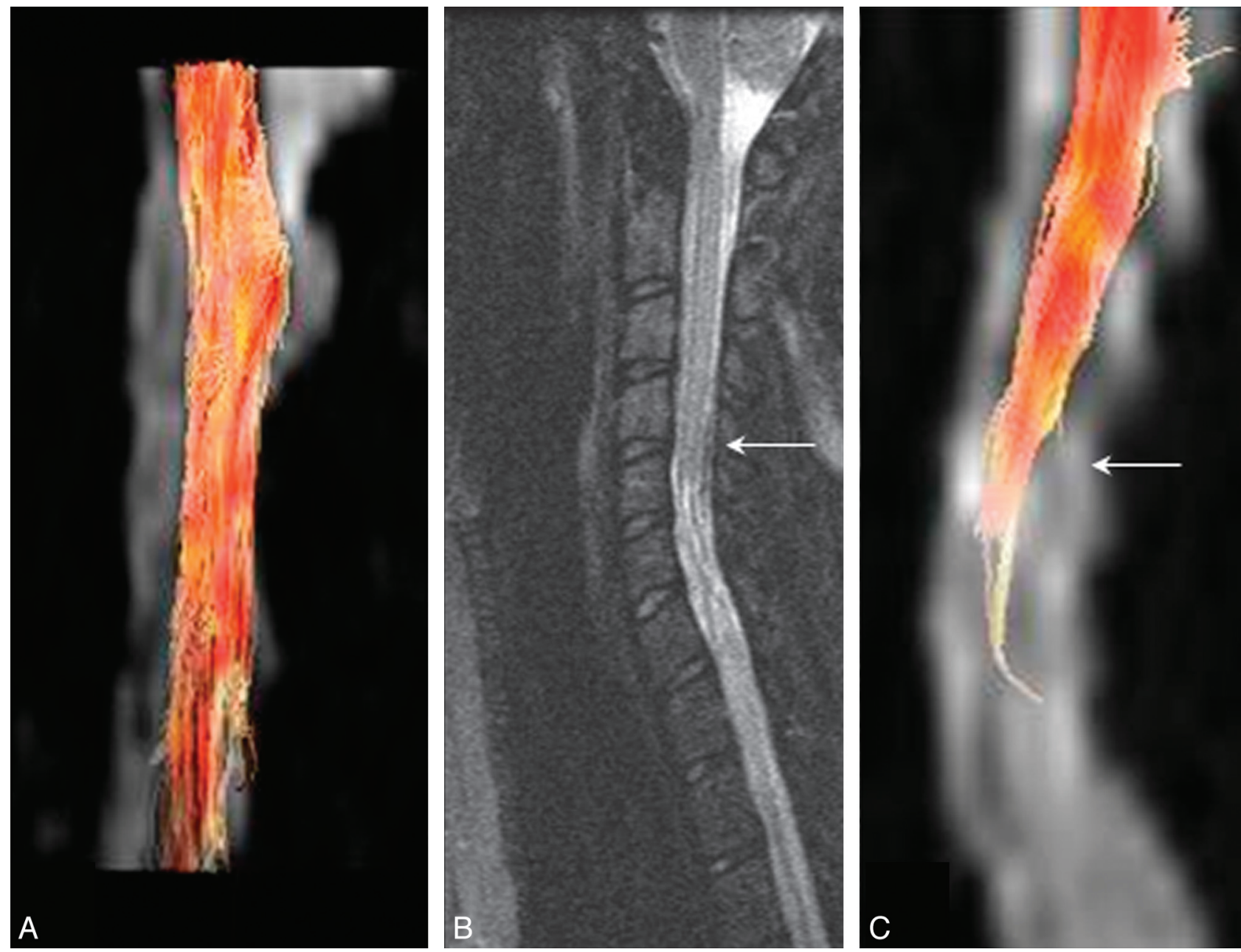

Fig 6. $A$, MR tractography images of the cervical spinal cord of a child with IS derived from FA values in the white matter tracts, a measure of degree of myelination of the white matter tracts along the spinal cord. $B$, Conventional midline sagittal T2-weighted image of a child with SCI (complete injury, ASIA A). C, An MR tractography image based on the FACT algorithm of the cervical spinal cord of the child in B. This algorithm failed, however, to track the rest of the cervical cord well below the injury (arrow) level, even though the FA measurements for this subject 4 showed recovery of FA values as seen in Fig 2.

\begin{tabular}{|c|c|c|}
\hline & $\begin{array}{c}\text { FA } r_{s} \\
(P \text { value }<.0001)^{\text {a }}\end{array}$ & $\begin{array}{c}\mathrm{D} r_{s} \\
(P \text { value }<.0001)^{\mathrm{a}}\end{array}$ \\
\hline PP score left & 0.28 & \\
\hline $\begin{array}{l}\text { Manual muscle test score, } \\
\text { upper extremities }\end{array}$ & -0.29 & 0.16 \\
\hline $\begin{array}{l}\text { Manual muscle test score, } \\
\text { lower extremities }\end{array}$ & 0.47 & -0.32 \\
\hline Total MS & 0.28 & \\
\hline $\mathrm{AC}$ & 0.50 & -0.33 \\
\hline Highest NL & & -0.26 \\
\hline LT score, right S4-5 & 0.50 & -0.24 \\
\hline LT score, left S4-5 & 0.50 & -0.26 \\
\hline LT total score, S4-5 & 0.50 & -0.25 \\
\hline PP score, right S4-5 & 0.50 & -0.27 \\
\hline PP score, left S4-5 & 0.50 & -0.29 \\
\hline Total PP, score S4-5 & 0.50 & -0.28 \\
\hline
\end{tabular}

a $P<.05$ is significant.

jury severity for both children and adults is under question. ${ }^{3,15}$ Without a reliable and definitive indication of the extent of injury, we are unable to provide families with prognosis or consistency in care, and we are unable to confidently attribute neurologic changes to treatment modalities. Even with older children in whom ISNCSCI have demonstrated strong reliability, an objective assessment of the cords is needed as an adjunct to the clinical examination. For example, with the eventual translation of laboratory-based therapies to the spinal cord, a method to evaluate treatment effectiveness on spinal tract injury is needed. Currently, various groups are studying pediatric models of SCI and investigating the utility of minimally invasive methods to introduce future therapies directly into the injured cord of children and restore neurologic function. As an adjunct to ISNCSCI, an objective method to evaluate treatment effectiveness at the cord level is needed. If DTI can be established as a reliable method of quantifying viable neural tissue within the spinal cord, it will be a critical neurodiagnostic adjunct to the clinical examination. Children with SCI routinely receive a standard MR imaging of the spine as per the standard of care, and performing DTI will add only approximately 8 minutes to the total imaging time.

The utility of DTI to measure spinal cord injury in the pediatric population has not been well studied. The pediatric population brings imaging challenges of a smaller FOV for the spinal cord and the possibility of increased motion in a notso-cooperative patient, thus potentially increasing the difficulty of obtaining accurate and reproducible DTI values. The 
difficulty is compounded by imaging factors such as volume averaging, motion artifacts from breathing and fluid pulsations, and susceptibility artifacts from the bones and lungs. These issues have been a limiting factor for studying the microstructure of the spinal cord by using DTI. In this study, we have compared DTI parameters of healthy spinal cord tissue in children with thoracolumbar IS with those of children with cervical SCI. This area of the spinal cord was chosen because it is less sensitive to motion and susceptibility artifacts. Assessment of the ability to detect DTI parameter differences in the pediatric population has allowed us to investigate the validity of DTI as a method to evaluate the consequence of SCI in children compared with concurrent clinical examinations and imaging techniques.

There are challenges to statistical analysis of the DTI parameters at different levels within the cord because of the locations of the SCIs and the varying heterogeneity of the severity of the injury within our subject population. Hence in this study, we chose to look at the entire cervical cord of the SCI subjects in comparison with that of children with IS. Future studies that include more children with SCI are required to evaluate the grade of injury by conventional T2 imaging and DTI scores at the level of injury as well as levels above and below the injured sites and correlations of these with ISNCSCI scores.

Preliminary FA and D values were determined for the pediatric population. The FA values seem to be similar to the values reported in adults, ${ }^{10,16,17}$ presumably due to the higher mean age of the healthy subject group. As with previously reported studies in adults, reduced FA and increased D values for injured spinal cord were seen in patients with SCI in comparison with healthy controls. Axial and radial diffusivities follow patterns similar to those of the average D measurements. As shown by other investigators, ${ }^{10}$ the greatest sensitivity is seen with the average $\mathrm{D}$ measurements. Test-retest reproducibility showed good correlation with thoracolumbar scoliosis and moderate reproducibility in subjects with SCI. While moderate correlation is acceptable for clinical instruments, the reason that stronger agreement was not demonstrated may be due to the variability of the level of cord injury among the subjects with SCI, because they were injured at different levels but their DTI indices were averaged across the entire cervical spinal cord. In future studies with a larger number of subjects, DTI measurements will be averaged and compared at every cord level. Previous studies have demonstrated variability of DTI indices due to cord location alone. ${ }^{8-10}$

The technical challenges of imaging the pediatric population, with the inherently smaller FOV and increased sensitivity to imaging artifacts such as motion, no doubt also contribute to the variability. The small FOV may exacerbate the inconsistency of the hand-drawn regions of interest for the diffusion parameter calculations, because the number of voxels in the FOV may be small, decreasing reproducibility of the DTI metrics. This was especially challenging in the subjects with SCI who had abnormal cord signals, thus making cord identification difficult. Furthermore, the failure of the FACT algorithm to track the rest of the cervical cord well below the injury level even though the FA measurements for the subject showed recovery of FA could be due to the following: It is known that tractography processes are susceptible to errors due to imag- ing noise causing inaccurate estimation of principal diffusion directions, modeling errors resulting from the microscopic anatomy of the white matter being more complex than can be represented by the choice of the model used (eg, crossing fibers and kissing fibers), and integration errors introduced in the tractography process. It is very likely that the lack of tracts in the lower part of the cord is presumably due to the contribution of $\geq 1$ of these errors.

There were statistically significant correlations between the various DTI indices and several ASIA clinical impairment scores (Table 2). Overall, there was a significant positive correlation between FA and ISNCSCI scores (left PP score, manual muscle test score of the lower extremities, total MS, AC, and total/individual [left and right] LT and PP scores for S4-5 dermatomes). In this study, it was found that higher FA values positively correlated with higher ISNCSCI scores, including AS in the S4-5 dermatomes and the presence of AC. Because FA measures the degree of myelination with higher FA values suggesting more intact spinal nerves, this positive correlation may be expected. In addition, it was found that lower D values negatively correlated with higher ISNCSCI scores, including manual muscle test scores of the lower extremities, AC, the highest NL, and total/individual (left and right) LT and PP scores for S4-5 dermatomes. Because D measures the degree of molecular water movement with lower values suggesting more intact spinal cord, this negative correlation may also be expected.

Before generalizing our findings of the positive FA and negative D correlation patterns found in this study and the strength of relationship between DTI and the ISNCSCI, additional studies with larger sample sizes are required. The subject with AIS D (motor incomplete) could theoretically have values similar to those in a typically developing subject due to the incompleteness of the injury that may have affected the correlation values obtained in this study. Also, the only fair relationship between DTI values and the manual muscle test score for the upper extremities may be due to the small sample size in this pilot study. Future studies will include a larger number of subjects with tetraplegia and paraplegia and levels of incompleteness, in which stratification for more detailed analysis will be possible.

One of the limitations of this study is the use of children with IS as the control group. Despite the fact that their spinal cords were healthy and their thoracolumbar scoliosis did not extend to the cervical skeletal region, the ideal control group would be typically developing children without any orthopedic or neurologic impairment. However, because the study was an initial feasibility study, we chose to use children who had an orthopedic impairment (thoracolumbar scoliosis) but no neurologic impairment (the cord was normal) and who were already scheduled for MR imaging as part of their clinical care. Future studies will include typically developing children. Another limitation of the study was the timing of the second scan. In any study of repeatability, the time interval between the first and subsequent testing interval should be long enough to avoid carryover or other influences of the first examination but short enough to ensure that change has not occurred. While the subjects in this study had chronic injuries and did not demonstrate changes in the clinical neurologic examination, the relatively long period ( 1 month) between the first and 
subsequent scan is a limitation because small changes in the cord could have potentially occurred. Ideally, in subjects with SCI, even chronic injuries, the time span between 2 scans should be minimized. Last, as with most pilot studies, the small number of subjects in this study is a limitation, as evidenced by the wide 95\% CI, and did not allow detailed analysis at individual spinal segments.

\section{Conclusions}

In this pilot study, we have shown that DTI measurements are feasible in children with and without SCI and show promising characteristics with respect to reliability. The differences in diffusion metrics between noninjured and injured spinal cords can be demonstrated in the pediatric population and may prove to be a more sensitive metric for spinal cord injury, as proposed by others in the literature for the adult population. Future work using reduced FOV imaging with $\mathrm{DTI}^{18}$ may reduce imaging artifacts to further improve the sensitivity and enable us to look at the entire spinal cord. These results are very encouraging and warrant further work with a large agematched cohort.

\section{References}

1. Mario RJ, Barros T, Biering-Sorensen F, et al. International standards for neurological classification of spinal cord injury. J Spinal Cord Med 2003;26(supp 1):50-56

2. Mulcahey MJ, Gaughan J, Betz RR, et al. The International Standards for Neurological Classification of Spinal Cord Injury: reliability of data when applied to children and youths. Spinal Cord 2007;45:452-59

3. Wietek $\mathrm{BM}$, Baron $\mathrm{CH}$, Hinnenghofen $\mathrm{H}$, et al. Cortical processing of residual anorectal sensation in patients with spinal cord injury: an FMRI study. Neurogastroenterol Motil 2008;20:488-97

4. Vogel LC, Sandani A, Chafetz R, et al. Intra-rater agreement of the anorectal exam and classification of injury severity in children with spinal cord injury. Spinal Cord 2009;47:687-91

5. Bonekamp D, Nagae LM, Degaonkar M, et al. Diffusion tensor imaging in children and adolescents: reproducibility, hemispheric, and age-related differences. Neuroimage 2007;34:733-42

6. Schwartz ED, Duda J, Shumsky JS, et al. Spinal cord diffusion tensor imaging and fiber tracking can identify white matter tract disruption and glial scar orientation following lateral funiculotomy. J Neurotrauma 2005;22:1388-98

7. Schwartz ED, Shumsky JS, Wehrli S, et al. Ex vivo MR determined apparent diffusion of coefficients correlate with motor recovery mediated by intraspinal transplants of fibroblasts genetically modified to express BDNF. Exp Neurol 2003;182:49-63

8. Mamata HM, De Girolami U, Hoge SH, et al. Collateral nerve fibers in human spinal cord: visualization with magnetic resonance diffusion tensor imaging. Neuroimage 2006;31:24-30

9. Mamata H, Jolesz FA, Maier SE. Apparent diffusion coefficient and fractional anisotropy in spinal cord: age and cervical spondylosis-related changes. $J$ Magn Reson Imaging 2005;22:38-43

10. Shanmuganathan K, Gullapalli RP, Zhuo J, et al. Diffusion tensor MR imaging in cervical spine trauma. AJNR Am J Neuroradiol 2008;29:655-59

11. Summers PE, Kewiecinski S, Staempfli P, et al. Human spinal cord diffusion tensor imaging at 3T. In: Proceedings of the 12th Scientific Meeting of the International Society of Magnetic Resonance in Medicine, Kyoto, Japan. May 5-21, 2004,

12. American Spinal Injury Association. Reference Manual for the International Standards for Neurological Classification of Spinal Cord Injury. Chicago: American Spinal Injury Association; 2003

13. Basser PJ, Pierpaoli C. A simplified method to measure the diffusion tensor from seven MR images. Magn Reson Med 1998;39:928-34

14. Behrens TE, Jbabdi S. MRI Diffusion Tractography. In: Johansen-Berg H, Behrens TE. Diffusion MRI: From Quantitative Measurement to In Vivo Neuroanatomy. London, UK: Academic Press; 2009:333-53

15. Samdani A, Fayssoux R, Asghar J, et al. Chronic spinal cord injury in the pediatric population: does magnetic resonance imaging correlate with the international standards for neurological classification of spinal cord injury? Spine 2009;34:74-81

16. Ellingson BM, Ulmer JL, Kurpad SN, et al. Diffusion tensor MR imaging of the neurologically intact human spinal cord. AJNR Am J Neuroradiol 2008;29:1279-84

17. Ellingson BM, Ulmer JL, Kurpad SN, et al. Diffusion tensor MR imaging in chronic spinal cord injury. AJNR Am J Neuroradiol 2008;29:1976-82

18. Finsterbusch J. High-resolution diffusion tensor imaging with inner field-ofview EPI. J Magn Reson Imaging 2009;29:987-93 